\title{
A STUDY ON LINEAR INEQUALITY REPRESENTATION OF SOCIAL WELFARE FUNCTIONS
}

\author{
Keisuke Sato \\ Railway Technical Research Institute
}

\author{
Yoshitsugu Yamamoto \\ University of Tsukuba
}

(Received December 20, 2007; Revised December 24, 2008)

\begin{abstract}
This paper presents a study on the recently proposed linear inequality representation of Arrovian Social Welfare Functions (ASWFs). We correct and show several sufficient conditions on preference domains for the linear inequalities of the representation to form integral polytopes. We also show that a given probabilistic ASWF induces a real vector satisfying the inequalities.
\end{abstract}

Keywords: Decision making, social welfare functions, Pareto principle, independence of irrelevant alternatives, linear inequality representation, integral polytope, extreme points.

\section{Introduction.}

Social choice theory discusses the way of aggregating individual opinions and making a decision of the society. Since Arrow [2] proved the classic Impossibility Theorem, this theory has been studied by various researchers of various academic fields. For the comprehension and the development of the theory see Arrow et al. [3] as well as Sen [11]. Recently Sethuraman et al. [12] formulated Arrovian Social Welfare Functions (ASWFs), which are social welfare functions satisfying fundamental Pareto principle and independence of irrelevant alternatives axioms, as integer solutions to a system of linear inequalities. They showed the Impossibility Theorem and other results such as Kalai and Muller [6] by solving their inequality system.

In this paper we study the linear inequality representation of ASWFs. We introduce a rather intuitive profile-dependent integer linear inequality representation of ASWFs and give a proof of the one-to-one correspondence result between an ASWF and an integer solution to the ASWF linear inequality representation in [12] using the profile-dependent one. Next we consider some polyhedral structure determined by the linear inequalities of the original ASWF representation. We give a counter-example to a claim in [12] about a sufficient condition on preference domains for the set of nonnegative solutions to the inequalities to form an integral polytope, and correct it by introducing "weakly nonisolated" condition about a triple of alternatives on the single-peaked domains. We also show that when the domain is single-caved or belongs to the ones on which each triple of alternatives contains an alternative that cannot be medium, and satisfies the weakly nonisolated condition, the integrality of the corresponding ASWF polytope is also guaranteed. We then discuss noninteger solutions to the inequality system. We show that a real vector satisfying the ASWF inequalities can be constructed from every "probabilistic" ASWF from the result about our profile-dependent representation, and then we derive a subadditive function of Barberá and Sonnenschein [4] as a special case. The construction of a probabilistic ASWF from a given 
solution to the inequalities is also studied.

The rest of the paper is organized as follows. Section 2 introduces notation and axioms. In Section 3 we introduce the ASWF integer linear inequality representation theorem of [12]. Discussions as to some polyhedral structure determined by the linear inequalities are held in Section 4. In Section 5 we discuss a relation between probabilistic ASWFs and noninteger solutions to the linear inequality representation. Section 6 concludes the paper and we give our view to future work.

\section{Notation and Axioms.}

The finite set of players is denoted by $\mathcal{N}=\{1,2, \ldots, n\}$ and we suppose that the number of players is at least two. Let $\mathcal{X}=\{x, y, z, \ldots\}$ be the set of finite number of alternatives and assume $|\mathcal{X}| \geq 3$. A binary relation $\succsim$ on $\mathcal{X}$ is a linear ordering if it satisfies

(i) completeness: $x \succsim y, y \succsim x$, or both hold for any pair of alternatives $x, y \in \mathcal{X}$,

(ii) transitivity: if $x \succsim y$ and $y \succsim z$ then $x \succsim z$ holds for any alternatives $x, y, z \in \mathcal{X}$, and

(iii) antisymmetry: if $x \succsim y$ and $y \succsim x$ then $x=y$ holds for any pair of alternatives $x, y \in \mathcal{X}$. When $x \succsim y$ but $y \succsim x$ we write $x \succ y$ and say " $x$ is (strictly) preferred to $y$." In the rest of the paper we discuss a pair or a triple of distinct alternatives so we use $\succ$ instead of $\succsim$. Let $L(\mathcal{X})$ be the set of all linear orderings on $\mathcal{X}$. We call a member of $L(\mathcal{X})$ a preference ordering or simply a preference. For a subset $\mathcal{Y} \subseteq \mathcal{X}$, we denote by $\succ \mid \mathcal{Y}$ the restriction of binary relation $\succ$ to $\mathcal{Y}$, i.e., $\left.\succ\right|_{\mathcal{Y}}$ is defined on $\mathcal{Y} \times \mathcal{Y}$ and $\left.x \succ\right|_{\mathcal{Y}} y$ if and only if $x \succ y$ and $x, y \in \mathcal{Y}$.

Let $\Omega$ be a nonempty subset of $L(\mathcal{X})$ and call it the preference domain or the domain. We set $\mathcal{P}=\Omega^{n}$, the $n$-ary Cartesian product of $\Omega$, call an element $p \in \mathcal{P}$ a profile, and denote by $\succ_{i}^{p}$ the preference of player $i$ at profile $p$. We denote by $\mathcal{S}(p, x \succ y)$ the set of players preferring alternative $x$ to $y$ at $p \in \mathcal{P}$, i.e.,

$$
\mathcal{S}(p, x \succ y):=\left\{i \in \mathcal{N} \mid x \succ_{i}^{p} y\right\} .
$$

Given $\mathcal{P} \subseteq L(\mathcal{X})^{n}$ we let $\mathcal{C}^{\mathcal{P}}(S, x \succ y)$ be the set of profiles at which only the players in $S$ prefer $x$ to $y$, that is,

$$
\mathcal{C}^{\mathcal{P}}(S, x \succ y):=\{p \in \mathcal{P} \mid \mathcal{S}(p, x \succ y)=S\}
$$

Let us define $\mathcal{N}^{\mathcal{P}}(x \succ y)$, which indicates whether the players can express their opinions as $x \succ y$ or not, and similarly $\mathcal{N}^{\mathcal{P}}(x \succ y \succ z)$, as

$$
\begin{aligned}
\mathcal{N}^{\mathcal{P}}(x \succ y): & = \begin{cases}\mathcal{N} & \text { if there exists a preference in } \Omega \text { such that } x \succ y, \\
\varnothing & \text { otherwise, }\end{cases} \\
\mathcal{N}^{\mathcal{P}}(x \succ y \succ z) & := \begin{cases}\mathcal{N} & \text { if there exists a preference in } \Omega \text { such that } x \succ y \succ z, \\
\varnothing & \text { otherwise, }\end{cases}
\end{aligned}
$$

for each distinct $x, y, z \in \mathcal{X}$ on a given $\mathcal{P}=\Omega^{n}$.

A social welfare function (on linear orderings) denoted by $f$, is a mapping that assigns an ordering on $\mathcal{X}$ to a profile $p \in \mathcal{P}$, i.e., $f: \mathcal{P} \rightarrow L(\mathcal{X})$. We denote by $\succ^{f(p)}$ the social preference ordering on $\mathcal{X}$ determined by $f$ at profile $p \in \mathcal{P}$. A social welfare function is said to satisfy unrestricted domain property when $\Omega=L(\mathcal{X})$ holds. If there exists a player $i \in \mathcal{N}$, such that $x \succ_{i}^{p} y$ implies $x \succ^{f(p)} y$ for any pair of distinct alternatives $x, y \in \mathcal{X}$ and for any profile $p \in \mathcal{P}$, then the function is said to be dictatorial and $i$ is said to be a 
dictator. A social welfare function satisfying the following two axioms is called an Arrovian Social Welfare Function (ASWF).

Definition 2.1 (Axiom of Pareto Principle (PP)). If the property that

$$
x \succ_{i}^{p} y \text { for all } i \in \mathcal{N} \text { implies } x \succ^{f(p)} y
$$

holds for any pair of distinct alternatives $x, y \in \mathcal{X}$ and for any profile $p \in \mathcal{P}$, then the social welfare function $f$ is said to have Pareto principle.

Definition 2.2 (Axiom of Independence of Irrelevant Alternatives (IIA)). If the property that

$$
\left.\succ_{i}^{p_{1}}\right|_{\{x, y\}}=\left.\succ_{i}^{p_{2}}\right|_{\{x, y\}} \text { for all } i \in \mathcal{N} \text { implies }\left.\succ^{f\left(p_{1}\right)}\right|_{\{x, y\}}=\left.\succ^{f\left(p_{2}\right)}\right|_{\{x, y\}}
$$

holds for any pair of distinct alternatives $x, y \in \mathcal{X}$ and for any pair of distinct profiles $p_{1}, p_{2} \in \mathcal{P}$, then the social welfare function $f$ is said to satisfy independence of irrelevant alternatives.

\section{Integer Linear Inequality Representation.}

This section introduces the linear inequality representation of ASWFs by [12] and the oneto-one correspondence theorem between an ASWF and a solution to the inequality system via a newly defined profile-dependent linear inequality representation of ASWFs. Suppose that ASWF $f$ is given. We introduce variable $d_{S}(x, y)$ for each pair of distinct alternatives $x, y \in \mathcal{X}$, and for each set $S$ such that $\mathcal{N}^{\mathcal{P}}(x \succ y) \backslash \mathcal{N}^{\mathcal{P}}(y \succ x) \subseteq S \subseteq \mathcal{N}^{\mathcal{P}}(x \succ y)$. The value of $d_{S}(x, y)$ is set by

$$
d_{S}(x, y):= \begin{cases}1 & \text { if } x \succ^{f(p)} y \text { holds for all } p \in \mathcal{C}^{\mathcal{P}}(S, x \succ y) \\ 0 & \text { otherwise }\end{cases}
$$

and we will give a certain integer linear inequality system to which the $d_{S}(x, y)$ constructed as above becomes a solution. On the other hand, for a given solution to this inequality system, we construct $f$, a function which maps a profile into a binary relation on $\mathcal{X}$ and is hopefully an ASWF, as follows: for each profile $p \in \mathcal{P}$ and for each pair of distinct alternatives $x, y \in \mathcal{X}$,

$$
\begin{aligned}
& x \succ^{f(p)} y \text { if } d_{\mathcal{S}(p, x \succ y)}(x, y)=1, \text { and } \\
& x \succ^{f(p)} y \text { otherwise. }
\end{aligned}
$$

We present below the inequality system with regard to $d_{S}(x, y)$.

Definition 3.1 (Integer Linear Inequality Representation of ASWFs).

(Integrality and IIA). For all pairs of distinct alternatives $x, y \in \mathcal{X}$, and for all sets $S$ such that $\mathcal{N}^{\mathcal{P}}(x \succ y) \backslash \mathcal{N}^{\mathcal{P}}(y \succ x) \subseteq S \subseteq \mathcal{N}^{\mathcal{P}}(x \succ y)$,

$$
d_{S}(x, y) \in\{0,1\} .
$$

(PP). For all pairs of distinct alternatives $x, y \in \mathcal{X}$ such that $\mathcal{N}^{\mathcal{P}}(x \succ y)=\mathcal{N}$,

$$
d_{\mathcal{N}}(x, y)=1 \text {. }
$$

(Completeness and Antisymmetry). For all pairs of distinct alternatives $x, y \in \mathcal{X}$, and for all sets $S$ such that $\mathcal{N}^{\mathcal{P}}(x \succ y) \backslash \mathcal{N}^{\mathcal{P}}(y \succ x) \subseteq S \subseteq \mathcal{N}^{\mathcal{P}}(x \succ y)$,

$$
d_{S}(x, y)+d_{\mathcal{N} \backslash S}(y, x)=1 .
$$


(Transitivity). For all ordered triples $(x, y, z)$ of distinct alternatives $x, y, z \in \mathcal{X}$, and for all sets $A \subseteq \mathcal{N}^{\mathcal{P}}(x \succ z \succ y), B \subseteq \mathcal{N}^{\mathcal{P}}(y \succ x \succ z), C \subseteq \mathcal{N}^{\mathcal{P}}(z \succ y \succ x), U \subseteq \mathcal{N}^{\mathcal{P}}(x \succ y \succ$ $z), V \subseteq \mathcal{N}^{\mathcal{P}}(z \succ x \succ y), W \subseteq \overline{\mathcal{N}}^{\mathcal{P}}(y \succ z \succ x)$, such that $(A, B, C, U, V, W)$ is a partition of $\mathcal{N}$

$$
d_{A \cup U \cup V}(x, y)+d_{B \cup U \cup W}(y, z)+d_{C \cup V \cup W}(z, x) \leq 2 .
$$

We note that there is a slight difference between the original ASWF formulation by [12] and that by Definition 3.1. For any pair of distinct $x, y \in \mathcal{X}$, the set $S$ takes between an empty set and the whole set $\mathcal{N}$ of players in the former, while $\mathcal{N}^{\mathcal{P}}(x \succ y) \backslash \mathcal{N}^{\mathcal{P}}(y \succ x) \subseteq S \subseteq$ $\mathcal{N}^{\mathcal{P}}(x \succ y)$ in the latter. The difference arises when $\mathcal{N}^{\mathcal{P}}(x \succ y)=\varnothing$ or $\mathcal{N}^{\mathcal{P}}(y \succ x)=\varnothing$, i.e., one alternative is always strictly preferred to the other for all preference orderings in the domain $\Omega$. This situation is said that either $(x, y)$ or $(y, x)$ is a trivial pair (see Definition 4.3). In [12] a constant value is set to the variables involving a trivial pair by convention, while we do not even enumerate such variables.

Theorem 3.2 (Sethuraman et al. [12], Theorem 1). Every solution to Integer Linear Inequality Representation of $A S W F$ s corresponds to an $A S W F$ on linear orderings and vice versa.

We give a proof of this theorem by introducing a profile-dependent variable $d(p, x, y)$ and a system of inequalities whose solution vector corresponds to an ASWF $f$ by the following natural way: for each $p \in \mathcal{P}$ and for each distinct $x, y \in \mathcal{X}$,

$$
\begin{aligned}
& x \succ^{f(p)} y \text { if and only if } d(p, x, y)=1, \text { and } \\
& x \nsucc^{f(p)} y \text { if and only if } d(p, x, y)=0 .
\end{aligned}
$$

Definition 3.3 (Profile-dependent Integer Linear Inequality Representation of ASWFs). (Integrality). For all profiles $p \in \mathcal{P}$, and for all pairs of distinct alternatives $x, y \in \mathcal{X}$,

$$
d(p, x, y) \in\{0,1\}
$$

(PP). For all profiles $p \in \mathcal{P}$, and for all pairs of distinct alternatives $x, y \in \mathcal{X}$, if $p \in \mathcal{C}^{\mathcal{P}}(\mathcal{N}, x \succ y)$ then

$$
d(p, x, y)=1
$$

(IIA). For all pairs of distinct profiles $p_{1}, p_{2} \in \mathcal{P}$, and for all pairs of distinct alternatives $x, y \in \mathcal{X}$, if $p_{1}, p_{2} \in \mathcal{C}^{\mathcal{P}}(S, x \succ y)$ for some $S$ then

$$
d\left(p_{1}, x, y\right)=d\left(p_{2}, x, y\right)
$$

(Completeness and Antisymmetry). For all profiles $p \in \mathcal{P}$, and for all pairs of distinct alternatives $x, y \in \mathcal{X}$,

$$
d(p, x, y)+d(p, y, x)=1 .
$$

(Transitivity). For all profiles $p \in \mathcal{P}$, and for all triples of distinct alternatives $x, y, z \in \mathcal{X}$,

$$
d(p, x, y)+d(p, y, z) \leq 1+d(p, x, z)
$$

*We say that $(A, B, C, U, V, W)$ is a partition of $\mathcal{N}$ if they are (possibly empty) disjoint sets whose union is $\mathcal{N}$. 


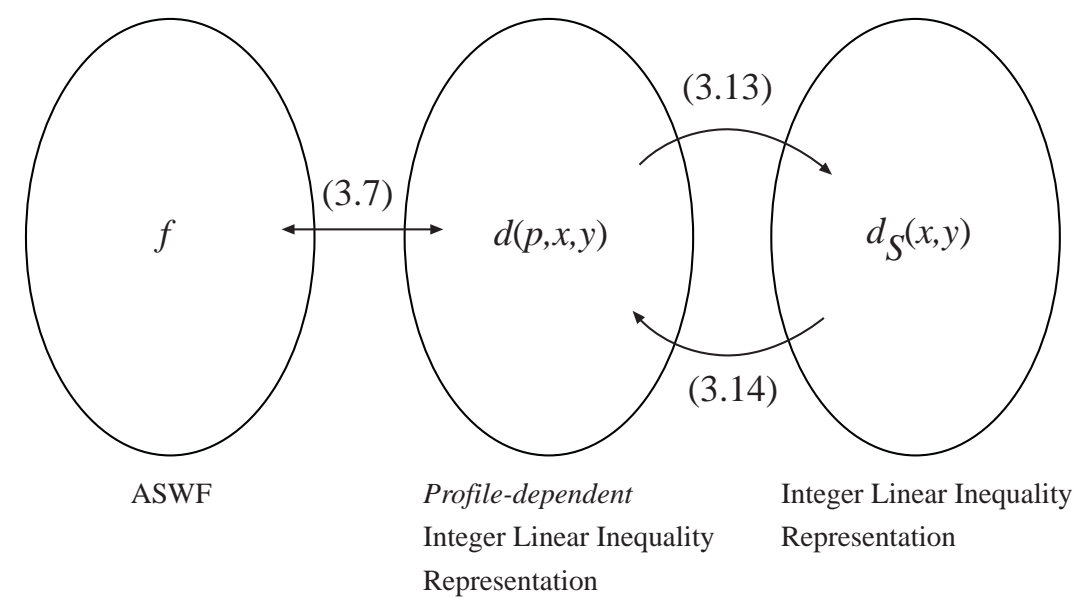

Figure 1: Correspondence among $f, d(p, x, y)$ and $d_{S}(x, y)$

Proof of Theorem 3.2. We connect the profile-dependent representation with the representation of Definition 3.1. Namely, let an arbitrary solution to (3.8)-(3.12) be given, and set $d_{S}(x, y)$ for each pair of distinct alternatives $x, y \in \mathcal{X}$, and for each set $S$ such that $\mathcal{N}^{\mathcal{P}}(x \succ y) \backslash \mathcal{N}^{\mathcal{P}}(y \succ x) \subseteq S \subseteq \mathcal{N}^{\mathcal{P}}(x \succ y)$ as

$$
d_{S}(x, y):=\frac{\sum_{p \in \mathcal{C}^{\mathcal{P}}(S, x \succ y)} d(p, x, y)}{\left|\mathcal{C}^{\mathcal{P}}(S, x \succ y)\right|} .
$$

In other words, we regard $d_{S}(x, y)$ as the ratio of $x$ being socially preferred to $y$ at the profiles such that all the players in $S$ express their preferences as $x \succ y$ and the rest as $y \succ x$. We confirm that $\mathcal{C}^{\mathcal{P}}(S, x \succ y)$ is not empty for each pair of distinct alternatives $x, y \in \mathcal{X}$ and for each set $S$ such that $\mathcal{N}^{\mathcal{P}}(x \succ y) \backslash \mathcal{N}^{\mathcal{P}}(y \succ x) \subseteq S \subseteq \mathcal{N}^{\mathcal{P}}(x \succ y)$. When $\mathcal{N}^{\mathcal{P}}(x \succ y)=\varnothing, S$ can only be $\varnothing$. In this case there does not exist a preference such that $x \succ y$. Therefore $S(p, x \succ y)=\varnothing$ holds for all $p \in \mathcal{P}$, and equivalently $\mathcal{C}^{\mathcal{P}}(\varnothing, x \succ y)=\mathcal{P}$. When $\mathcal{N}^{\mathcal{P}}(x \succ y)=\mathcal{N}$ and $\mathcal{N}^{\mathcal{P}}(y \succ x)=\varnothing, S$ can only be $\mathcal{N}$. This means that for all profiles every player strictly prefers $x$ to $y$, thus $\mathcal{C}^{\mathcal{P}}(\mathcal{N}, x \succ y) \neq \varnothing$ holds. Each player can express $x \succ y$ or $y \succ x$ when both $\mathcal{N}^{\mathcal{P}}(x \succ y)$ and $\mathcal{N}^{\mathcal{P}}(y \succ x)$ are $\mathcal{N}$, so in this case $\mathcal{C}^{\mathcal{P}}(S, x \succ y)$ is nonempty for any $\varnothing \subseteq S \subseteq \mathcal{N}$. From this discussion we see that $d_{S}(x, y)$ constructed by (3.13) is well-defined. Meanwhile, given a solution to Integer Linear Inequality Representation of ASWFs in the sense of Definition 3.1, we create $d(p, x, y)$ for each $p \in \mathcal{P}$ and for each pair of distinct alternatives $x, y \in \mathcal{X}$ as follows:

$$
d(p, x, y):=d_{\mathcal{S}(p, x \succ y)}(x, y) .
$$

Since $p \in \mathcal{C}^{\mathcal{P}}(S, x \succ y)$ holds if and only if $\mathcal{S}(p, x \succ y)=S$ holds, $d(p, x, y)$ obtained from a given $d_{S}(x, y)$ by $(3.14)$ equals $d_{S}(x, y)$ by (3.13). The converse also holds if (3.10) is assumed. Hence it suffices to show that a solution to (3.8)-(3.12) constructs a solution to (3.3)-(3.6) and a given $d_{S}(x, y)$ gives $d(p, x, y)$ which satisfies the constraints (3.8)-(3.12) in Definition 3.3.

Let an arbitrary solution to (3.8)-(3.12) be given. We take an arbitrary pair of distinct $x, y \in \mathcal{X}$, and an arbitrary but appropriate $S$. From (3.10) and (3.13), the equation

$$
d_{S}(x, y)=d(p, x, y)
$$


holds for any $p \in \mathcal{C}^{\mathcal{P}}(S, x \succ y)$. This equation along with (3.8) yields (3.3). Consider the case where $S=\mathcal{N}^{\mathcal{P}}(x \succ y)=\mathcal{N}$, and we have (3.4) from (3.9). When a profile $p$ is in $\mathcal{C}^{\mathcal{P}}(S, x \succ y)$ it is also a member of $\mathcal{C}^{\mathcal{P}}(\mathcal{N} \backslash S, y \succ x)$ because of the antisymmetry of a linear preference ordering. Therefore, (3.11) and (3.15) imply

$$
d_{S}(x, y)+d_{\mathcal{M} \backslash S}(y, x)=d(p, x, y)+d(p, y, x)=1,
$$

which is (3.5). To show that (3.6) is satisfied, suppose the contrary. Then for some distinct $x, y, z \in \mathcal{X}$ and for some partition $(A, B, C, U, V, W)$ of $\mathcal{N}$ with $A \subseteq \mathcal{N}^{\mathcal{P}}(x \succ z \succ y)$, $B \subseteq \mathcal{N}^{\mathcal{P}}(y \succ x \succ z), C \subseteq \mathcal{N}^{\mathcal{P}}(z \succ y \succ x), U \subseteq \mathcal{N}^{\mathcal{P}}(x \succ y \succ z), V \subseteq \mathcal{N}^{\mathcal{P}}(z \succ x \succ y)$, and $W \subseteq \mathcal{N}^{\mathcal{P}}(y \succ z \succ x)$

$$
d_{A \cup U \cup V}(x, y)+d_{B \cup U \cup W}(y, z)+d_{C \cup V \cup W}(z, x)>2
$$

holds. If player $i$ is in $A$, there exists a preference ordering on which $i$ can express his/her opinion as $x \succ z \succ y$. This is because $i \in A$ implies $\mathcal{N}^{\mathcal{P}}(x \succ z \succ y)=\mathcal{N}$, the existence of a preference with $x \succ z \succ y$. Likewise $i \in B$ means that $i$ can choose a preference from $\Omega$ which is $y \succ x \succ z$, etc. Note also that each player is in exactly one of $A, B, C, U, V$, and $W$, and that we can choose a profile $p \in \mathcal{P}$ such that, for each $i \in \mathcal{N}$,

if $i \in A$ then $x \succ_{i}^{p} z \succ_{i}^{p} y$, else if $i \in C$ then $z \succ_{i}^{p} y \succ_{i}^{p} x$, else if $i \in V$ then $z \succ_{i}^{p} x \succ_{i}^{p} y$, else if $i \in B$ then $y \succ_{i}^{p} x \succ_{i}^{p} z$, else if $i \in U$ then $x \succ_{i}^{p} y \succ_{i}^{p} z$, else if $i \in W$ then $y \succ_{i}^{p} z \succ_{i}^{p} x$.

For this profile, we have $p \in \mathcal{C}^{\mathcal{P}}(A \cup U \cup V, x \succ y), p \in \mathcal{C}^{\mathcal{P}}(B \cup U \cup W, y \succ z)$, and $p \in \mathcal{C}^{\mathcal{P}}(C \cup V \cup W, z \succ x)$ hold. Then by (3.15), we have

$$
d(p, x, y)+d(p, y, z)+d(p, z, x)>2 .
$$

On the other hand by applying (3.11) to (3.12) we obtain

$$
d(p, x, y)+d(p, y, z)+d(p, z, x) \leq 2
$$

a contradiction.

Let a solution to Integer Linear Inequality Representation of ASWFs in the sense of Definition 3.1 be given, and the value of $d(p, x, y)$ be set by (3.14). We show that this $d(p, x, y)$ satisfies (3.8)-(3.12). The constructed $d(p, x, y)$ in this way satisfies (3.10) since the statement $p_{1}, p_{2} \in \mathcal{C}^{\mathcal{P}}(S, x \succ y)$ is equivalent to $\mathcal{S}\left(p_{1}, x \succ y\right)=\mathcal{S}\left(p_{2}, x \succ y\right)=S$. In the case where $S=\mathcal{N},(3.9)$ is implied by (3.4). The set $\mathcal{S}(p, x \succ y)$ lies between $\mathcal{N}^{\mathcal{P}}(x \succ y) \backslash \mathcal{N}^{\mathcal{P}}(y \succ x)$ and $\mathcal{N}^{\mathcal{P}}(x \succ y)$, hence we have (3.8) from (3.3). Antisymmetry of preference orderings in the common preference domain framework guarantees $\mathcal{S}(p, y \succ x)=$ $\mathcal{N} \backslash \mathcal{S}(p, x \succ y)$, and this together with (3.5) assures us

$$
d(p, x, y)+d(p, y, x)=d_{\mathcal{S}(p, x \succ y)}(x, y)+d_{\mathcal{S}(p, y \succ x)}(y, x)=1,
$$

which is (3.11). Let us suppose that (3.12) does not hold for some $p \in \mathcal{P}$ and for some distinct alternatives $x, y, z \in \mathcal{X}$. Then (3.16) is also violated by these $p, x, y$, and $z$, i.e.,

$$
d(p, x, y)=1, d(p, y, z)=1, \text { and } d(p, z, x)=1 .
$$

It means, by (3.14), that

$$
d_{\mathcal{S}(p, x \succ y)}(x, y)=1, d_{\mathcal{S}(p, y \succ z)}(y, z)=1 \text {, and } d_{\mathcal{S}(p, z \succ x)}(z, x)=1,
$$


respectively. Regarding the triple $\{x, y, z\}$, there are six patterns of preference orderings at this $p$. We partition the whole set $\mathcal{N}$ of players into the six subsets as follows:

$$
\begin{array}{ll}
\bar{A}:=\left\{i \in N \mid x \succ_{i}^{p} z \succ_{i}^{p} y\right\}, & \bar{B}:=\left\{i \in N \mid y \succ_{i}^{p} x \succ_{i}^{p} z\right\}, \\
\bar{C}:=\left\{i \in N \mid z \succ_{i}^{p} y \succ_{i}^{p} x\right\}, & \bar{U}:=\left\{i \in N \mid x \succ_{i}^{p} y \succ_{i}^{p} z\right\}, \\
\bar{V}:=\left\{i \in N \mid z \succ_{i}^{p} x \succ_{i}^{p} y\right\}, & \bar{W}:=\left\{i \in N \mid y \succ_{i}^{p} z \succ_{i}^{p} x\right\} .
\end{array}
$$

Since $\mathcal{S}(p, x \succ y)=\bar{A} \cup \bar{U} \cup \bar{V}, \mathcal{S}(p, y \succ z)=\bar{B} \cup \bar{U} \cup \bar{W}$, and $\mathcal{S}(p, z \succ x)=\bar{C} \cup \bar{V} \cup \bar{W}$ hold, we have

$$
d_{\bar{A} \cup \bar{U} \cup \bar{V}}(x, y)+d_{\bar{B} \cup \bar{U} \cup \bar{W}}(y, z)+d_{\bar{C} \cup \bar{V} \cup \bar{W}}(z, x)=3 .
$$

This contradicts (3.6) because the sets $\bar{A}, \bar{B}, \bar{C}, \bar{U}, \bar{V}$, and $\bar{W}$ are all disjoint, their union coincides with $\mathcal{N}$, and $\bar{A}$ is a subset of $\mathcal{N}^{\mathcal{P}}(x \succ z \succ y), \bar{B} \subseteq \mathcal{N}^{\mathcal{P}}(y \succ x \succ z)$, etc.

Now we see the one-to-one correspondence between an ASWF and a solution to Integer Linear Inequality Representation of ASWFs under the transformations (3.7), (3.13) and (3.14).

When (3.5) is assumed, the transitivity inequalities (3.6) 'for all "ordered" triples' can be substituted for those 'for all "unordered" triples' by evaluating all permutations of every triple, which contributes to a reduction in the number of constraints.

Definition 3.4 (Transitivity on Unordered Triples). For all unordered triples $\{x, y, z\}$ of distinct alternatives $x, y, z \in \mathcal{X}$, and for all sets $A \subseteq \mathcal{N}^{\mathcal{P}}(x \succ z \succ y), B \subseteq \mathcal{N}^{\mathcal{P}}(y \succ x \succ z)$, $C \subseteq \mathcal{N}^{\mathcal{P}}(z \succ y \succ x), U \subseteq \mathcal{N}^{\mathcal{P}}(x \succ y \succ z), V \subseteq \mathcal{N}^{\mathcal{P}}(z \succ x \succ y), W \subseteq \mathcal{N}^{\mathcal{P}}(y \succ z \succ x)$, such that $(A, B, C, U, V, W)$ is a partition of $\mathcal{N}$,

$$
1 \leq d_{A \cup U \cup V}(x, y)+d_{B \cup U \cup W}(y, z)+d_{C \cup V \cup W}(z, x) \leq 2
$$

\section{Polyhedral Structure of Linear Inequalities.}

This section studies some polyhedral structure determined by the linear inequalities (3.4)(3.6) along with the nonnegativity of the variables. We introduce the weakly nonisolated condition on the preference domain and show that all the extreme points of the polytope are integers when the domain is single-peaked, single-caved, or the domain on which each triple of alternatives contains an alternative that cannot be medium, and it satisfies the weakly nonisolated condition.

Definition 4.1 (Single-peakedness). Let $\triangleright$ be a linear ordering on $\mathcal{X}$, i.e., $\triangleright \in L(\mathcal{X})$. The domain $\Omega$ is called single-peaked with respect to $\triangleright$ if $\Omega$ is a subset of $\Omega^{P}(\triangleright)$ defined as

$\Omega^{P}(\triangleright):=\{\succ \in L(\mathcal{X}) \mid$ for every distinct $x, y, z \in \mathcal{X}$ with $x \triangleright y \triangleright z, y \succ x$ or $y \succ z$ holds. $\}$

We refer to the linear ordering $\triangleright$ as the reference linear ordering.

The class of single-peaked domains is well known and seen for example in $[2,11]$. Concerning the polyhedral structure on single-peaked domains the following is claimed in [12]. Claim [Sethuraman et al. [12], Theorem 10] When $\Omega$ is single-peaked (with respect to $\triangleright \in L(\mathcal{X})$ ) the set of nonnegative solutions satisfying (3.4)-(3.6) is an integral polytope, i.e., all the extreme points are integer vectors.

We discuss here an example of the ASWF linear inequality formulation and its polytope on a single-peaked domain. 
Example 4.2. Let $n=2, \mathcal{X}=\{u, v, w, x, y, z\}, \Omega=\{u \succ v \succ w \succ x \succ y \succ z, z \succ y \succ$ $x \succ w \succ v \succ u\}$, and the reference linear ordering $\triangleright$ be $u \triangleright v \triangleright w \triangleright x \triangleright y \triangleright z$. The domain $\Omega$ is obviously single-peaked with respect to $\triangleright$. We show below its ASWF linear inequalities (3.4), (3.5), and (3.17) that determine a polytope. Note that we choose here an unordered triple $\{a, b, c\}$ with $a \triangleright b \triangleright c$ to enumerate all the inequalities of (3.17) for arbitrary distinct alternatives $a, b, c \in \mathcal{X}$. The sets $A, B, V$, and $W$ in (3.17) must always be empty since only the preferences with $a \succ b \succ c$ and $c \succ b \succ a$ are admissible.

For all pairs of distinct alternatives $a, b \in \mathcal{X}$,

$$
d_{\mathcal{N}}(a, b)=1, d_{\varnothing}(a, b)=0, d_{\{2\}}(a, b)=1-d_{\{1\}}(b, a) .
$$

For all unordered triple $\{a, b, c\}$ of alternatives $a, b, c \in \mathcal{X}$ such that $a \triangleright b \triangleright c$, and for all disjoint sets $U, C$ such that $C \cup U=\mathcal{N}$,

$$
1 \leq d_{U}(a, b)+d_{U}(b, c)+d_{C}(c, a) \leq 2 .
$$

For this system of inequalities,

$$
\begin{array}{lllll}
d_{\{1\}}(u, v)=0.5, & d_{\{1\}}(u, w)=0, & d_{\{1\}}(u, x)=0.5, & d_{\{1\}}(u, y)=0.5, & d_{\{1\}}(u, z)=0, \\
d_{\{1\}}(v, u)=0, & d_{\{1\}}(v, w)=0, & d_{\{1\}}(v, x)=0.5, & d_{\{1\}}(v, y)=0, & d_{\{1\}}(v, z)=0.5, \\
d_{\{1\}}(w, u)=0, & d_{\{1\}}(w, v)=0, & d_{\{1\}}(w, x)=0.5, & d_{\{1\}}(w, y)=0.5, & d_{\{1\}}(w, z)=0.5, \\
d_{\{1\}}(x, u)=0, & d_{\{1\}}(x, v)=0, & d_{\{1\}}(x, w)=0, & d_{\{1\}}(x, y)=0, & d_{\{1\}}(x, z)=0, \\
d_{\{1\}}(y, u)=0, & d_{\{1\}}(y, v)=0, & d_{\{1\}}(y, w)=0, & d_{\{1\}}(y, x)=0, & d_{\{1\}}(y, z)=0.5, \\
d_{\{1\}}(z, u)=0, & d_{\{1\}}(z, v)=0, & d_{\{1\}}(z, w)=0, & d_{\{1\}}(z, x)=0, & d_{\{1\}}(z, y)=0, \\
\text { and } d_{\mathcal{N}}(a, b)=1, d_{\varnothing}(a, b)=0, & d_{\{2\}}(a, b)=1-d_{\{1\}}(b, a)
\end{array}
$$

for all pairs of distinct alternatives $a, b \in \mathcal{X}$,

is a feasible solution and is seen to be the unique solution to the following equalities:

$$
\begin{aligned}
& d_{\{1\}}(u, w)=0, \quad d_{\{1\}}(u, z)=0, \quad d_{\{1\}}(v, u)=0, \quad d_{\{1\}}(v, w)=0, \quad d_{\{1\}}(v, y)=0, \\
& d_{\{1\}}(w, u)=0, \quad d_{\{1\}}(w, v)=0, \quad d_{\{1\}}(x, u)=0, \quad d_{\{1\}}(x, v)=0, \quad d_{\{1\}}(x, w)=0, \\
& d_{\{1\}}(x, y)=0, \quad d_{\{1\}}(x, z)=0, \quad d_{\{1\}}(y, u)=0, \quad d_{\{1\}}(y, v)=0, \quad d_{\{1\}}(y, w)=0, \\
& d_{\{1\}}(y, x)=0, \quad d_{\{1\}}(z, u)=0, \quad d_{\{1\}}(z, v)=0, \quad d_{\{1\}}(z, w)=0, \quad d_{\{1\}}(z, x)=0, \\
& d_{\{1\}}(z, y)=0 \text {, } \\
& d_{\{1\}}(u, v)+d_{\{1\}}(v, y)+d_{\{2\}}(y, u)=1, d_{\{1\}}(u, w)+d_{\{1\}}(w, x)+d_{\{2\}}(x, u)=1, \\
& d_{\{1\}}(u, w)+d_{\{1\}}(w, y)+d_{\{2\}}(y, u)=1, d_{\{1\}}(u, x)+d_{\{1\}}(x, y)+d_{\{2\}}(y, u)=1, \\
& d_{\{1\}}(v, w)+d_{\{1\}}(w, x)+d_{\{2\}}(x, v)=1, d_{\{1\}}(v, w)+d_{\{1\}}(w, z)+d_{\{2\}}(z, y)=1 \text {, } \\
& d_{\{1\}}(v, x)+d_{\{1\}}(x, z)+d_{\{2\}}(z, v)=1 \text {, } \\
& d_{\{1\}}(u, v)+d_{\{1\}}(v, z)+d_{\{2\}}(z, u)=2, d_{\{1\}}(u, y)+d_{\{1\}}(y, z)+d_{\{2\}}(z, u)=2 \text {, } \\
& \text { and } d_{\mathcal{N}}(a, b)=1, d_{\varnothing}(a, b)=0, d_{\{2\}}(a, b)=1-d_{\{1\}}(b, a) \\
& \text { for all pairs of distinct alternatives } a, b \in \mathcal{X} \text {. }
\end{aligned}
$$

Thus the fractional solution given by (4.2) is an extreme point of the polytope determined by the linear inequality system (4.1). This is a counter-example to Theorem 10 in [12].

We impose an additional condition on a single-peaked domain and make the polytope integral. For the introduction of the new condition let us present the commonly known concept of a trivial pair of alternatives on admissible preferences, and then we define triviality on an unordered triple of distinct alternatives.

Definition 4.3 (Trivial Pair). An ordered pair $(x, y)$ of distinct alternatives $x, y \in \mathcal{X}$ is said to be a trivial pair on $\Omega$ if $x \succ y$ holds for all preference orderings in $\Omega$.

Definition 4.4 (Triviality over an Unordered Triple). For an unordered triple of distinct alternatives $x, y, z \in \mathcal{X}$, we say that $\{x, y, z\}$ has a trivial pair on $\Omega$ if at least one of $(x, y)$, $(y, x),(x, z),(z, x),(y, z)$, and $(z, y)$ is a trivial pair. 
Then we refer to the notion of an isolated triple, which is originally proposed by [12], and define nonisolation on an unordered triple.

Definition 4.5 (Isolated Triple). An ordered triple $(x, y, z)$ of distinct alternatives $x, y, z \in$ $\mathcal{X}$, is said to be an isolated triple on $\Omega$ if there exists a preference ordering in $\Omega$ with $x \succ y \succ z$ and there does not exist preference ordering in $\Omega$ with $y \succ z \succ x$ or $z \succ x \succ y$.

Definition 4.6 (Nonisolation over an Unordered Triple). For an unordered triple of distinct alternatives $x, y, z \in \mathcal{X}$, we say that $\{x, y, z\}$ has nonisolation on $\Omega$ if none of $(x, y, z)$, $(y, z, x),(z, x, y),(x, z, y),(z, y, x)$, and $(y, x, z)$ is an isolated triple.

From the above definitions we finally introduce the condition on the preference domain that we call weak nonisolation and show the corrected version of Theorem 10 in [12].

Definition 4.7 (Weak Nonisolation). The domain $\Omega$ is said to be weakly nonisolated if every unordered triple in $\mathcal{X}$ has either a trivial pair or nonisolation on $\Omega$.

Note that the domain $\Omega$ in Example 4.2 is not weakly nonisolated because $(x, y, z)$ is an isolated triple while there is no trivial pair on $\Omega$.

Theorem 4.8. When $\Omega$ is single-peaked with respect to the reference linear ordering $\square \in$ $L(\mathcal{X})$ and weakly nonisolated, the set of nonnegative solutions satisfying (3.4)-(3.6) is an integral polytope.

Proof. We follow the technique of proof in [12]: given an arbitrary nonnegative solution to the linear inequalities, we round it to an integer by a certain procedure, show that the rounded solution still satisfies the inequalities, and see that these facts guarantee the integrality of the polytope. Let $\mathcal{O}^{\mathcal{P}}$ be the polytope that we are to discuss, that is, the set of nonnegative solutions satisfying (3.4), (3.5), and (3.17) instead of (3.6) on the given $\mathcal{P}=\Omega^{n}$. Let $d$ be an arbitrary vector of $d_{S}(x, y)$ 's with $d \in \mathcal{O}^{\mathcal{P}}$. We generate a random number $Z$ from the uniform distribution between 0 and 1 . We round the vector $d$ to a $0-1$ vector, say $d^{\prime}$, by the following way: for each pair of distinct alternatives $a, b \in \mathcal{X}$, and for each $S$ with $\mathcal{N}^{\mathcal{P}}(a \succ b) \backslash \mathcal{N}^{\mathcal{P}}(b \succ a) \subseteq S \subseteq \mathcal{N}^{\mathcal{P}}(a \succ b)$,

$$
\text { if } a \triangleright b, d_{S}^{\prime}(a, b):=\left\{\begin{array}{ll}
1 & \text { if } d_{S}(a, b)>Z, \\
0 & \text { otherwise }
\end{array} \text { else, } d_{S}^{\prime}(a, b):= \begin{cases}1 & \text { if } d_{S}(a, b) \geq 1-Z, \\
0 & \text { otherwise }\end{cases}\right.
$$

We see that $d^{\prime}$ trivially satisfies (3.4) for any $Z$. Because $d_{S}(x, y)+d_{\mathcal{N} \backslash S}(y, x)=1$ for an arbitrary pair of distinct alternatives $x, y \in \mathcal{X}$ and for an arbitrary but appropriate $S$, $d_{S}(x, y)>Z$ holds if and only if $d_{\mathcal{N} \backslash S}(y, x)<1-Z$ holds and $d_{S}(x, y) \leq Z$ if and only if $d_{\mathcal{N} \backslash S}(y, x) \geq 1-Z$. Either one of $d_{S}(x, y)$ or $d_{\mathcal{N} \backslash S}(y, x)$ is 1 and the other is 0 when rounded, which means that (3.5) is also satisfied for $d^{\prime}$.

Take an arbitrary triple of distinct alternatives $x, y, z \in \mathcal{X}$ with $x \triangleright y \triangleright z$, and consider (3.17) for $\{x, y, z\}$. By the single-peakedness of the domain with respect to $\triangleright$, there does not exist a preference in $\Omega$ such that $x \succ z \succ y$ or $z \succ x \succ y$. The sets $\mathcal{N}^{\mathcal{P}}(x \succ z \succ y)$ and $\mathcal{N}^{\mathcal{P}}(z \succ x \succ y)$ are empty, and (3.17) for $\{x, y, z\}$ can be written without the sets $A$ and $V$ : for all sets $B \subseteq \mathcal{N}^{\mathcal{P}}(y \succ x \succ z), C \subseteq \mathcal{N}^{\mathcal{P}}(z \succ y \succ x), U \subseteq \mathcal{N}^{\mathcal{P}}(x \succ y \succ z)$, $W \subseteq \mathcal{N}^{\mathcal{P}}(y \succ z \succ x)$, such that $(B, C, U, W)$ is a partition of $\mathcal{N}$,

$$
1 \leq d_{U}(x, y)+d_{B \cup U \cup W}(y, z)+d_{C \cup W}(z, x) \leq 2 .
$$

Case 1: $\{x, y, z\}$ has a trivial pair. When $(x, y)$ is trivial, there does not exist a preference with $y \succ x$, which indicates that $\mathcal{N}^{\mathcal{P}}(y \succ x \succ z), \mathcal{N}^{\mathcal{P}}(z \succ y \succ x)$, and $\mathcal{N}^{\mathcal{P}}(y \succ z \succ x)$ are all empty. Since $(B, C, U, W)$ is a partition of $\mathcal{N}, U$ must be $\mathcal{N}$, and we have $d_{U}(x, y)=1$, 
$d_{B \cup U \cup W}(y, z)=1$, and $d_{C \cup W}(z, x)=d_{\varnothing}(z, x)=0$ from (3.4) and (3.5). Then the rounded solution satisfies (4.3), for 1 is rounded to 1 and 0 to 0 for any $Z$.

When $(y, x)$ is trivial, $\mathcal{N}^{\mathcal{P}}(x \succ y \succ z)=\varnothing$, and $d_{U}(x, y)=d_{\varnothing}(x, y)=0$. Then the rounded solution $d^{\prime}$ trivially satisfies the "less than or equal to 2 " part of (4.3). The rest is to show that

$$
1 \leq d_{B \cup W}(y, z)+d_{C \cup W}(z, x)
$$

is satisfied even after $d$ is rounded. We see that if $d_{B \cup W}(y, z) \leq Z$, then $d_{C \cup W}(z, x) \geq 1-Z$. Therefore $d^{\prime}$ satisfies this inequality from the rounding rule as well as $y \triangleright z$ and $x \triangleright z$.

When $(x, z)$ is trivial, $\mathcal{N}^{\mathcal{P}}(z \succ y \succ x)$ and $\mathcal{N}^{\mathcal{P}}(y \succ z \succ x)$ are the empty set. Accordingly $B \cup U=\mathcal{N}$ holds. This indicates $d_{B \cup U \cup W}(y, z)=1$ and $d_{C \cup W}(z, x)=0$, so the rounded $d^{\prime}$ clearly satisfies (4.3).

When $(z, x)$ is trivial, it means $\mathcal{N}^{\mathcal{P}}(y \succ x \succ z)=\mathcal{N}^{\mathcal{P}}(x \succ y \succ z)=\varnothing$. Then $d_{U}(x, y)=0$, and $d_{C \cup W}(z, x)=1$ due to $C \cup W=\mathcal{N}$. In this case $d^{\prime}$ satisfies (4.3) again.

When $(y, z)$ is trivial, $C \subseteq \mathcal{N}^{\mathcal{P}}(z \succ y \succ x)=\varnothing$, and $d_{B \cup U \cup W}(y, z)=1$ since $B \cup U \cup W$ must be $\mathcal{N}$. The rounded solution trivially satisfies the "greater than or equal to 1 " part of (4.3). The rest is to prove that

$$
d_{U}(x, y)+d_{W}(z, x) \leq 1
$$

is kept satisfied after the rounding procedure. We see that if $d_{U}(x, y)>Z$, then $d_{W}(z, x)<$ $1-Z$. Hence by $x \triangleright y$ and $x \triangleright z$ this inequality is satisfied for $d^{\prime}$.

When $(z, y)$ is trivial, $\mathcal{N}^{\mathcal{P}}(y \succ x \succ z), \mathcal{N}^{\mathcal{P}}(x \succ y \succ z)$, and $\mathcal{N}^{\mathcal{P}}(y \succ z \succ x)$ are empty. This means $B=U=W=\varnothing$ and $C=\mathcal{N}$. Accordingly we obtain $d_{U}(x, y)=$ $d_{B \cup U \cup W}(y, z)=0$ and $d_{C \cup W}(z, x)=1$. The variables do not change if rounded.

Case 2: $\{x, y, z\}$ does not have a trivial pair. From the single-peakedness of $\Omega$ there is not a preference with $z \succ x \succ y$ or $x \succ z \succ y$. Then there exist preferences in $\Omega$ with $x \succ y \succ z$, $z \succ y \succ x$, respectively, otherwise $(y, x)$ or $(y, z)$ is trivial. Since $\{x, y, z\}$ is nonisolated, a preference with $y \succ z \succ x$ exists in $\Omega$, and also a preference such that $y \succ x \succ z$. Therefore, $\mathcal{N}^{\mathcal{P}}(y \succ x \succ z), \mathcal{N}^{\mathcal{P}}(z \succ y \succ x), \mathcal{N}^{\mathcal{P}}(x \succ y \succ z)$, and $\mathcal{N}^{\mathcal{P}}(y \succ z \succ x)$ are all equal to $\mathcal{N}$, and (4.3) holds for any partition $(B, C, U, W)$ of $\mathcal{N}$. Take an arbitrary partition $(B, C, U, W)$, and let $W^{\prime}=C \cup W, C^{\prime}=\varnothing$. Because $\left(B, C^{\prime}, U, W^{\prime}\right)$ is also a partition of $\mathcal{N}$, $d$ satisfies the following:

$$
\begin{aligned}
2 \geq d_{U}(x, y)+d_{B \cup U \cup W^{\prime}}(y, z)+d_{C^{\prime} \cup W^{\prime}}(z, x) & =d_{U}(x, y)+d_{B \cup U \cup C \cup W}(y, z)+d_{C \cup W}(z, x) \\
& =d_{U}(x, y)+1+d_{C \cup W}(z, x) .
\end{aligned}
$$

By rounding $d_{U}(x, y)$ and $d_{C \cup W}(z, x)$ in this inequality, we have

$$
d_{U}^{\prime}(x, y)+d_{C \cup W}^{\prime}(z, x) \leq 1
$$

from the rounding procedure together with $x \triangleright y$ and $x \triangleright z$. We see that $d^{\prime}$ satisfies the "less than or equal to $2 "$ part of (4.3), whether $d_{B \cup U \cup W}(y, z)$ is rounded to 1 or 0 . Regarding the

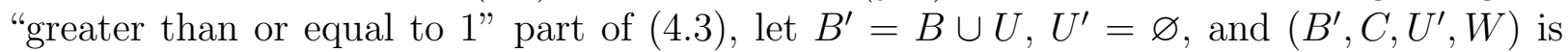
also a partition of $\mathcal{N}$. Hence,

$$
1 \leq d_{U^{\prime}}(x, y)+d_{B^{\prime} \cup U^{\prime} \cup W}(y, z)+d_{C \cup W}(z, x)=d_{B \cup U \cup W}(y, z)+d_{C \cup W}(z, x)
$$

holds. If we round the vector $d$ in this inequality, then

$$
d_{B \cup U \cup W}^{\prime}(y, z)+d_{C \cup W}^{\prime}(z, x) \geq 1
$$


is obtained. This observation implies that $d^{\prime}$ satisfies the "greater than or equal to 1" part of (4.3) regardless of the rounded value of $d_{U}(x, y)$. Hence we have confirmed that for any $d \in \mathcal{O}^{\mathcal{P}}$ the rounded vector $d^{\prime}$ is also in $\mathcal{O}^{\mathcal{P}}$.

Now we are ready to consider our main problem. Suppose that the polytope $\mathcal{O}^{\mathcal{P}}$, the set of nonnegative solutions which satisfies (3.4), (3.5), and (3.17), has an extreme point which is not an integer. Here we let $\bar{d}$ be such a point. Since $\bar{d}$ is an extreme point of $\mathcal{O}^{\mathcal{P}}$ there exists a cost vector $c$ such that $\bar{d}$ becomes the unique optimal solution to the linear programming problem:

$$
\mid \begin{array}{ll}
\operatorname{minimize} & c^{\top} d \\
\text { subject to } & d \in \mathcal{O}^{\mathcal{P}}
\end{array}
$$

where $c^{\top}$ denotes the transpose of $c$. We generate a random number $Z$ and round $\bar{d}$ by the procedure proposed above. The rounded vector, denoted by $\bar{d}^{\prime}$, is still in $\mathcal{O}^{\mathcal{P}}$ as we have discussed. Now $\bar{d}$ is assumed to be the unique optimal solution to $(\mathrm{P})$, then

$$
c^{\top} \bar{d}^{\prime}>c^{\top} \bar{d}
$$

holds for any $Z$. Meanwhile, the expected value of each component of $\overline{d^{\prime}}$ is

$$
\begin{aligned}
\text { if } a \triangleright b, & E\left(\bar{d}_{S}^{\prime}(a, b)\right)=0 \times P\left(Z \geq \bar{d}_{S}(a, b)\right)+1 \times P\left(Z<\bar{d}_{S}(a, b)\right)=\bar{d}_{S}(a, b), \\
\text { else, } & E\left(\bar{d}_{S}^{\prime}(a, b)\right)=0 \times P\left(Z<1-\bar{d}_{S}(a, b)\right)+1 \times P\left(Z \geq 1-\bar{d}_{S}(a, b)\right)=\bar{d}_{S}(a, b),
\end{aligned}
$$

for each $a, b \in \mathcal{X}$ and for each $S$ which lies between $\mathcal{N}^{\mathcal{P}}(a \succ b) \backslash \mathcal{N}^{\mathcal{P}}(b \succ a)$ and $\mathcal{N}^{\mathcal{P}}(a \succ b)$, since $Z$ is uniformly distributed between 0 and 1 . This fact implies

$$
E\left(c^{\top} \bar{d}^{\prime}\right)=c^{\top} \bar{d}
$$

contradicting (4.4). Thus all the extreme points of $\mathcal{O}^{\mathcal{P}}$ are integers.

We give another type of preference domains, called single-caved domains, and show that such domains along with weakly nonisolation condition also form integral polytopes.

Definition 4.9 (Single-cavedness). The domain $\Omega$ is called single-caved with respect to $\triangleright \in L(\mathcal{X})$ if $\Omega$ is a subset of $\Omega^{C}(\triangleright)$ defined as

$\Omega^{C}(\triangleright):=\{\succ \in L(\mathcal{X}) \mid$ for every distinct $x, y, z \in \mathcal{X}$ with $x \triangleright y \triangleright z, x \succ y$ or $z \succ y$ holds. $\}$

Theorem 4.10. When $\Omega$ is single-caved with respect to $\triangleright \in L(\mathcal{X})$ and weakly nonisolated, the set of nonnegative solutions satisfying (3.4)-(3.6) is an integral polytope.

Proof. The same proof technique applies to this case as the case where $\Omega$ is single-peaked, so we omit the proof.

Single-peakedness (with respect to some reference ordering) is interpreted as that at each player's admissible preference a certain one in any triple of distinct alternatives cannot be the worst among the three, while single-cavedness is that a certain one in any triple cannot be the best. Both of them are treated as special cases of Value Restriction (VR) by Sen $[10,11]$. Another example of VR referred to in [10] is that one alternative cannot be medium among three alternatives. We derive integral polytopes on the domains that have such property.

Definition 4.11 (Cannot-be-medium Property). For an ordered triple $(x, y, z)$ of distinct alternatives $x, y, z \in \mathcal{X}$, it is said that $y$ cannot be medium among $(x, y, z)$ on $\Omega$ if there does not exist a preference ordering in $\Omega$ such that $x \succ y \succ z$ or $z \succ y \succ x$. 
Theorem 4.12. When each triple of alternatives contains an alternative that cannot be medium on $\Omega$ and $\Omega$ is weakly nonisolated, the set of nonnegative solutions satisfying (3.4)(3.6) is an integral polytope.

Proof. Again we apply the same technique as when $\Omega$ is single-peaked, except that the rounding rule is changed as follows: for each pair of distinct alternatives $a, b \in \mathcal{X}$, and for each $S$ with $\mathcal{N}^{\mathcal{P}}(a \succ b) \backslash \mathcal{N}^{\mathcal{P}}(b \succ a) \subseteq S \subseteq \mathcal{N}^{\mathcal{P}}(a \succ b)$,

$$
\text { if } 1 \in S, d_{S}^{\prime}(a, b):=\left\{\begin{array}{ll}
1 & \text { if } d_{S}(a, b)>Z, \\
0 & \text { otherwise }
\end{array} \text { else, } d_{S}^{\prime}(a, b):= \begin{cases}1 & \text { if } d_{S}(a, b) \geq 1-Z \\
0 & \text { otherwise }\end{cases}\right.
$$

Given an arbitrary vector $d \in \mathcal{O}^{\mathcal{P}}$, the rounded $d^{\prime}$ clearly satisfies (3.4) for any $Z$, and also (3.5) since player 1 is always a member of either $S$ or $\mathcal{N} \backslash S$ but not both. Now it suffices to show that $d^{\prime}$ satisfies (3.17). Let us take an arbitrary triple of distinct alternatives $x, y, z \in \mathcal{X}$, and consider (3.17) for $\{x, y, z\}$ with $y$ cannot be medium. Then $\mathcal{N}^{\mathcal{P}}(x \succ y \succ$ $z)=\mathcal{N}^{\mathcal{P}}(z \succ y \succ x)=\varnothing$ holds, and (3.17) for $\{x, y, z\}$ reduces to this: for all sets $A \subseteq \mathcal{N}^{\mathcal{P}}(x \succ z \succ y), B \subseteq \mathcal{N}^{\mathcal{P}}(y \succ x \succ z), V \subseteq \mathcal{N}^{\mathcal{P}}(z \succ x \succ y), W \subseteq \mathcal{N}^{\mathcal{P}}(y \succ z \succ x)$, such that $(A, B, V, W)$ is a partition of $\mathcal{N}$,

$$
1 \leq d_{A \cup V}(x, y)+d_{B \cup W}(y, z)+d_{V \cup W}(z, x) \leq 2 .
$$

Case 1: $\{x, y, z\}$ has a trivial pair. When $(x, y)$ is trivial, $B$ and $W$ must be empty and $A \cup V=\mathcal{N}$ holds. Then $d_{A \cup V}(x, y)=d_{\mathcal{N}}(x, y)=1$ as well as $d_{B \cup W}(y, z)=d_{\varnothing}(y, z)=0$ holds. Recall that 1 is rounded to 1 and 0 to 0 for any $Z$, and we see that the rounded $d^{\prime}$ satisfies (4.5) regardless of the value of $d_{V \cup W}^{\prime}(z, x)$.

When $(y, x)$ is trivial, $A=V=\varnothing$ and the union of $B$ and $W$ is the whole set of players. This time $d_{A \cup V}(x, y)=0$ and $d_{B \cup W}(y, z)=1$ hold. Again (4.5) is still satisfied after $d$ is rounded.

When $(x, z)$ is trivial, both $\mathcal{N}^{\mathcal{P}}(z \succ x \succ y)$ and $\mathcal{N}^{\mathcal{P}}(y \succ z \succ x)$ are empty, and accordingly $V$ and $W$ are. Then $d_{V \cup W}(z, x)=0$ and the rounded $d^{\prime}$ trivially satisfies the "less than or equal to 2" part of (4.5). We round the rest inequality:

$$
1 \leq d_{A}(x, y)+d_{B}(y, z) .
$$

Player 1 is in exactly either $A$ or $B$, so we see that $d^{\prime}$ still satisfies this inequality.

When $(z, x)$ is trivial, it means $A=B=\varnothing$ and $d_{V \cup W}(z, x)=d_{\mathcal{N}}(z, x)=1$. Hence the "greater than or equal to 1 " part of (4.5) is cleared, and

$$
d_{V}(x, y)+d_{W}(y, z) \leq 1
$$

is still satisfied after the rounding, for player 1 is a member of either $V$ or $W$ but not both.

When $(y, z)$ is trivial, $\mathcal{N}^{\mathcal{P}}(x \succ z \succ y)$ and $\mathcal{N}^{\mathcal{P}}(z \succ x \succ y)$ are empty. The set of players $\mathcal{N}$ is partitioned into $B$ and $W$, which is the same case as $(y, x)$-trivial.

When $(z, y)$ is trivial, $B \subseteq \mathcal{N}^{\mathcal{P}}(y \succ x \succ z)=\varnothing$ and $W \subseteq \mathcal{N}^{\mathcal{P}}(y \succ z \succ x)=\varnothing$. For the rest of sets it holds that $A \cup V=\mathcal{N}$. This is the same situation where $(x, y)$ is trivial.

Case 2: $\{x, y, z\}$ does not have a trivial pair. The cannot-be-medium property of $y$ means that there is not a preference with $x \succ y \succ z$ or $z \succ y \succ x$ in $\Omega$. Then it can be said that there exist preferences such that $x \succ z \succ y, y \succ x \succ z, z \succ x \succ y$, and $y \succ z \succ x$. That is because an isolated triple emerges if any one of them is removed. Thus 
(4.5) holds for any partition $(A, B, V, W)$ of $\mathcal{N}$. Take an arbitrary partition $(A, B, V, W)$, and let $V^{\prime}=A \cup V, W^{\prime}=B \cup W, A^{\prime}=B^{\prime}=\varnothing$. We see that $\left(A^{\prime}, B^{\prime}, V^{\prime}, W^{\prime}\right)$ is a partition of $\mathcal{N}$. Then $d$ satisfies the following:

$$
2 \geq d_{A^{\prime} \cup V^{\prime}}(x, y)+d_{B^{\prime} \cup W^{\prime}}(y, z)+d_{V^{\prime} \cup W^{\prime}}(z, x)=d_{A \cup V}(x, y)+d_{B \cup W}(y, z)+1 .
$$

We round $d_{A \cup V}(x, y)$ and $d_{B \cup W}(y, z)$ in this inequality, and obtain

$$
d_{A \cup V}^{\prime}(x, y)+d_{B \cup W}^{\prime}(y, z) \leq 1
$$

since player 1 is either in $A \cup V$ or in $B \cup W$. This inequality implies that the "less than or equal to 2" part of (4.5) is satisfied at $d^{\prime}$. With regard to the "greater than or equal to 1" part of (4.5), let $A^{\prime}=A \cup V, V^{\prime}=B \cup W, V^{\prime}=W^{\prime}=\varnothing$, and we have a partition $\left(A^{\prime}, B^{\prime}, V^{\prime}, W^{\prime}\right)$ of the set of the players. Then $d$ satisfies the following:

$$
1 \leq d_{A^{\prime} \cup V^{\prime}}(x, y)+d_{B^{\prime} \cup W^{\prime}}(y, z)+d_{V^{\prime} \cup W^{\prime}}(z, x)=d_{A \cup V}(x, y)+d_{B \cup W}(y, z) .
$$

We round the $d$ in this inequality and obtain

$$
d_{A \cup V}^{\prime}(x, y)+d_{B \cup W}^{\prime}(y, z) \geq 1
$$

This observation tells us that $d^{\prime}$ satisfies the "greater than or equal to 1" part of (4.5).

We have confirmed that for a given $d \in \mathcal{O}^{\mathcal{P}}$, the rounded vector $d^{\prime}$ is also in $\mathcal{O}^{\mathcal{P}}$. Then assuming that there is a noninteger extreme point leads to a contradiction as we have seen in the single-peaked case.

\section{Probabilistic ASWFs and Linear Inequality Representation.}

In this section we discuss the relation between a noninteger solution to the linear inequality representation and a probabilistic social welfare function. A probability measure on $L(\mathcal{X})$ is a function $\ell: 2^{L(\mathcal{X})} \rightarrow[0,1]$ such that $\ell(\varnothing)=0, \ell(L(\mathcal{X}))=1$, and $\ell\left(\omega_{1} \cup \omega_{2}\right)=$ $\ell\left(\omega_{1}\right)+\ell\left(\omega_{2}\right)-\ell\left(\omega_{1} \cap \omega_{2}\right)$ for all $\omega_{1}, \omega_{2} \in 2^{L(\mathcal{X})}$. We let $\mathcal{L}(L(\mathcal{X}))$ be the set of all probability measures on $L(\mathcal{X})$. We introduce here a probabilistic social welfare function of [4] which gives each linear ordering on $\mathcal{X}$ a certain probability of its occurrence when a profile is input. Definition 5.1. A probabilistic social welfare function (on linear orderings) is a mapping, say $h$, that maps each profile to a probability measure on $L(\mathcal{X})$, that is, $h: \mathcal{P} \rightarrow \mathcal{L}(L(\mathcal{X}))$, where $\mathcal{P}=\Omega^{n} \subseteq L(\mathcal{X})^{n}$. The probabilistic social welfare function $h$ is said to satisfy unrestricted domain property when $\Omega=L(\mathcal{X})$.

Let $p \in \mathcal{P}$ be a profile, then $h(p)$ is a probability measure on $L(\mathcal{X})$. Given distinct alternatives $x, y \in \mathcal{X}$, consider the set $\left\{\triangleright \in L(\mathcal{X}) \mid \triangleright_{\{x, y\}}=x \succ y\right\}$. We define $r^{h}(p, x \succ$ $y$ ) as the sum of probabilities that $h$ gives to the preference orderings such that $x \succ y$, i.e.,

$$
r^{h}(p, x \succ y):=h(p)\left(\left\{\triangleright \in L(\mathcal{X}) \mid \triangleright_{\{x, y\}}=x \succ y\right\}\right) .
$$

We call the function $h$ satisfying the probabilistic version of Arrow's two axioms shown below a probabilistic Arrovian Social Welfare Function (probabilistic ASWF for short).

Definition 5.2 (Axiom of Probabilistic Pareto Principle (PPP)). If the property that

$$
x \succ_{i}^{p} y \text { for all } i \in \mathcal{N} \text { implies } r^{h}(p, x \succ y)=1
$$

holds for any pair of distinct alternatives $x, y \in \mathcal{X}$ and for any profile $p \in \mathcal{P}$, then the probabilistic social welfare function $h$ is said to have the Pareto principle. 
Definition 5.3 (Axiom of Probabilistic Independence of Irrelevant Alternatives (PIIA)). If the property that

$$
\left.\succ_{i}^{p_{1}}\right|_{\{x, y\}}=\left.\succ_{i}^{p_{2}}\right|_{\{x, y\}} \text { for all } i \in \mathcal{N} \text { implies } r^{h}\left(p_{1}, x \succ y\right)=r^{h}\left(p_{2}, x \succ y\right)
$$

holds for any pair of distinct alternatives $x, y \in \mathcal{X}$ and for any pair of distinct profiles $p_{1}, p_{2} \in \mathcal{P}$, then the probabilistic social welfare function $h$ is said to satisfy independence of irrelevant alternatives.

We show that a given probabilistic ASWF induces a real vector satisfying the inequalities of the ASWF representation and it derives a subadditive function of [4] on the family of subsets of players under unrestricted domain property. The vector is constructed in the following way: given an $h: \mathcal{P} \rightarrow \mathcal{L}(L(\mathcal{X}))$, we set

$$
d_{S}(x, y):=\frac{\sum_{p \in \mathcal{C}^{\mathcal{P}}(S, x \succ y)} r^{h}(p, x \succ y)}{\left|\mathcal{C}^{\mathcal{P}}(S, x \succ y)\right|}
$$

for each pair of distinct alternatives $x, y \in \mathcal{X}$ and for each set $S$ such that $\mathcal{N}^{\mathcal{P}}(x \succ y) \backslash$ $\mathcal{N}^{\mathcal{P}}(y \succ x) \subseteq S \subseteq \mathcal{N}^{\mathcal{P}}(x \succ y)$.

Theorem 5.4. For every probabilistic $A S W F, d_{S}(x, y)$ defined by (5.1) is a nonnegative solution to (3.4)-(3.6).

Proof. Note that

$$
\left\{\triangleright \in L(\mathcal{X}) \mid \triangleright_{\{x, y\}}=x \succ y\right\} \cup\left\{\triangleright \in L(\mathcal{X}) \mid \triangleright_{\{x, y\}}=y \succ x\right\}=L(\mathcal{X}),
$$

and we see from the definition of $r^{h}$ that

$$
r^{h}(p, x \succ y)+r^{h}(p, y \succ x)=1
$$

and

$$
\begin{aligned}
r^{h}(p, x \succ y)+r^{h}(p, y \succ z) & =r^{h}(p, x \succ y \text { or } y \succ z)+r^{h}(p, x \succ y \text { and } y \succ z) \\
& \leq 1+r^{h}(p, x \succ z)
\end{aligned}
$$

hold for any profile $p \in \mathcal{P}$ and for any distinct $x, y, z \in \mathcal{X}$. We simply let

$$
d(p, x, y):=r^{h}(p, x \succ y)
$$

then (3.11) as well as (3.12) in Section 3 is satisfied. We also see that (3.9) holds because of Axiom (PPP), and (3.10) holds because of Axiom (PIIA). As we have seen in the proof of Theorem 3.2 that $d_{S}(x, y)$ defined by $d(p, x, y)$ through (3.13) satisfies (3.4)-(3.6), $d_{S}(x, y)$ defined by (5.1) satisfies (3.4)-(3.6).

Note that under Axiom (PIIA) $d_{S}(x, y)$ of (5.1) satisfies

$$
d_{S}(x, y)=r^{h}(p, x \succ y) \quad \text { for any } p \in \mathcal{C}^{\mathcal{P}}(S, x \succ y)
$$

or equivalently

$$
d_{\mathcal{S}(p, x \succ y)}(x, y)=r^{h}(p, x \succ y) .
$$

While the following statement can be found in [12], we give the proof to make this paper more self-contained. 
Lemma 5.5. When the domain is unrestricted, the solution $d_{S}(x, y)$ to (3.4)-(3.6) does not depend on the pair $(x, y)$ of alternatives.

Proof. We consider (3.6) for an arbitrary triple of distinct alternatives $x, y, z \in \mathcal{X}$. By unrestricted domain property, $\mathcal{N}^{\mathcal{P}}(x \succ z \succ y), \mathcal{N}^{\mathcal{P}}(y \succ x \succ z), \mathcal{N}^{\mathcal{P}}(z \succ y \succ x), \mathcal{N}^{\mathcal{P}}(x \succ$ $y \succ z), \mathcal{N}^{\mathcal{P}}(z \succ x \succ y)$, and $\mathcal{N}^{\mathcal{P}}(y \succ z \succ x)$ are all $\mathcal{N}$. Take an arbitrary $S \subseteq \mathcal{N}$ and let $U=S, W=\mathcal{N} \backslash S$, the other sets be empty. Then the following holds:

$$
d_{S}(x, y)+d_{S \cup(\mathcal{N} \backslash S)}(y, z)+d_{\mathcal{N} \backslash S}(z, x) \leq 2 .
$$

Applying (3.4) and (3.5) to this inequality ensures

$$
d_{S}(x, y) \leq d_{S}(x, z)
$$

Meanwhile, we set $U=S, V=\mathcal{N} \backslash S$ and have

$$
d_{S}(y, z) \leq d_{S}(x, z)
$$

Exchanging $x, y$ and $z$ we have

$$
d_{S}(x, y) \leq d_{S}(x, z) \leq d_{S}(y, z) \leq d_{S}(y, x) \leq d_{S}(z, x) \leq d_{S}(z, y) \leq d_{S}(x, y),
$$

which means that the value of $d_{S}(x, y)$ does not depend on the pair of alternatives but only the set $S$ for $\{x, y, z\}$. If we repeat the procedure for all triples of alternatives we complete the proof.

Thus, when the domain is unrestricted, the system (3.4)-(3.6) reduces to

$$
\begin{array}{ll}
d_{\mathcal{N}}=1, & \\
d_{S}+d_{\mathcal{N} \backslash S}=1 & \text { for each } S \subseteq \mathcal{N}, \\
d_{A \cup U \cup V}+d_{B \cup U \cup W}+d_{C \cup V \cup W} \leq 2 & \text { for each partition }(A, B, C, U, V, W) \text { of } \mathcal{N} .
\end{array}
$$

Now we are ready to prove the following theorem via the linear inequality representation of ASWFs.

Theorem 5.6 (Barberá and Sonnenschein [4]). For every probabilistic ASWF $h$ on the unrestricted domain, there exists a function $\mu: 2^{\mathcal{N}} \rightarrow \mathbb{R}$ such that

$$
\mu(\mathcal{S}(p, x \succ y))=r^{h}(p, x \succ y)
$$

for each profile $p \in \mathcal{P}$ and for each pair of distinct alternatives $x, y \in \mathcal{X}$, and furthermore $\mu$ satisfies

$$
\begin{array}{ll}
\mu(\mathcal{N})=1, & \text { for each } S \subseteq \mathcal{N}, \\
\mu(S)+\mu(\mathcal{N} \backslash S)=1 & \text { for each } S, T \subseteq \mathcal{N} \text { (subadditivity). }
\end{array}
$$

Proof. Suppose $d$ satisfies (5.4)-(5.6) and let $S$ and $T$ be arbitrary subsets of $\mathcal{N}$. We set $C=S \cap T, U=\mathcal{N} \backslash(S \cup T), V=T \backslash S$ and $W=S \backslash T$. Then we see

$$
d_{S \cup T} \leq d_{S}+d_{T}
$$

Since we have seen (5.3), we simply let

$$
\mu(S):=d_{S}
$$


Note that the following monotonicity condition in the original version of Theorem 5.6:

$$
\mu\left(S^{\prime}\right) \leq \mu(S) \text { for each } S, S^{\prime} \subseteq \mathcal{N} \text { with } S^{\prime} \subseteq S,
$$

can be derived from (5.8)-(5.10) by setting $T=\mathcal{N} \backslash S^{\prime}$ as follows:

$$
\mu(S)+\mu\left(\mathcal{N} \backslash S^{\prime}\right) \geq \mu\left(S \cup\left(\mathcal{N} \backslash S^{\prime}\right)\right)=\mu(\mathcal{N})=1=\mu\left(S^{\prime}\right)+\mu\left(\mathcal{N} \backslash S^{\prime}\right)
$$

We next consider the construction of a probabilistic ASWF from a solution to the linear inequalities. Let $\mathcal{H}^{\mathcal{P}}$ be the set of all probabilistic ASWFs on the given $\mathcal{P}=\Omega^{n} \subseteq L(\mathcal{X})^{n}$, and recall that $\mathcal{O}^{\mathcal{P}}$ defined in the proof of Theorem 4.8 is the set of nonnegative solutions satisfying (3.4)-(3.6). We denote by $\Gamma^{\mathcal{P}}: \mathcal{H}^{\mathcal{P}} \rightarrow \mathcal{O}^{\mathcal{P}}$ a function that assigns a nonnegative solution in $\mathcal{O}^{\mathcal{P}}$ to a probabilistic ASWF by (5.1). Concerning its characteristics, the first question is whether $\Gamma^{\mathcal{P}}$ is injective. The following example shows that it is not injective in general.

Example 5.7. Let $n=2, \mathcal{X}=\{x, y, z\}$, and $\Omega=\{x \succ y \succ z, z \succ y \succ x\}$. Then there are four profiles, named $p_{1}, \ldots, p_{4}$. We define two probabilistic ASWFs $h_{1}, h_{2}$ where the probability of each social preference being selected at each profile is shown in Table 1. While these two functions $h_{1}$ and $h_{2}$ are different, they give the same value of $r^{h}$ and hence $d_{S}(x, y)$. In fact, take $p_{2}$ for example, then we have

$$
r^{h_{1}}\left(p_{2}, x \succ y\right)=h_{1}\left(p_{2}\right)(\{x \succ y \succ z, x \succ z \succ y, z \succ x \succ y\})=\frac{1}{2}+0+0=\frac{1}{2},
$$

and

$$
r^{h_{2}}\left(p_{2}, x \succ y\right)=h_{1}\left(p_{2}\right)(\{x \succ y \succ z, x \succ z \succ y, z \succ x \succ y\})=\frac{1}{4}+\frac{1}{4}+0=\frac{1}{2} .
$$

The second question is whether $\Gamma^{\mathcal{P}}$ is surjective, i.e., if there is a probabilistic ASWF for each point of $\mathcal{O}^{\mathcal{P}}$. Concerning the problem, we should observe the result of McLennan [7] that when the number of alternatives is six or more and the domain is unrestricted, the function $\mu$ in Theorem 5.6 is additive, that is,

$$
\mu(S \cup T)=\mu(S)+\mu(T) \text { for all disjoint } S, T \subseteq \mathcal{N}
$$

is also satisfied in addition to (5.8)-(5.10). Using this result we see by the next example that $\Gamma^{\mathcal{P}}$ is not surjective in general.

Example 5.8. Let $n \geq 3,|\mathcal{X}| \geq 6$, and $\mathcal{P}=\Omega^{n}=L(\mathcal{X})^{n}$, i.e., unrestricted domain. For each pair of distinct alternatives $x, y \in \mathcal{X}$, and for each set $S \subseteq \mathcal{N}$, we set

$$
d_{S}(x, y):= \begin{cases}1 & \text { if } S=\mathcal{N} \\ \frac{1}{2} & \text { if } \varnothing \subsetneq S \subsetneq \mathcal{N} \\ 0 & \text { if } S=\varnothing .\end{cases}
$$

This is clearly a fractional solution to (3.4)-(3.6). Suppose that this is obtained from a probabilistic ASWF $h$ through (5.1). Then $\mu(S)=d_{S}(x, y)$ from Lemma 5.5 and equations (5.3) and (5.7). Equation (5.11) is violated, however, when we partition $\mathcal{N}$ into three nonempty sets $\left(S_{1}, S_{2}, S_{3}\right)$ and observe that $\mu\left(S_{1}\right)+\mu\left(S_{2}\right)=1$ whereas $\mu\left(S_{1} \cup S_{2}\right)=\mu(\mathcal{N} \backslash$ $\left.S_{3}\right)=1 / 2$. Hence $d_{S}(x, y)$ is not an image of $\Gamma^{\mathcal{P}}$. 
Table 1: Two different probabilistic ASWFs that yield the same $d_{S}(x, y)$

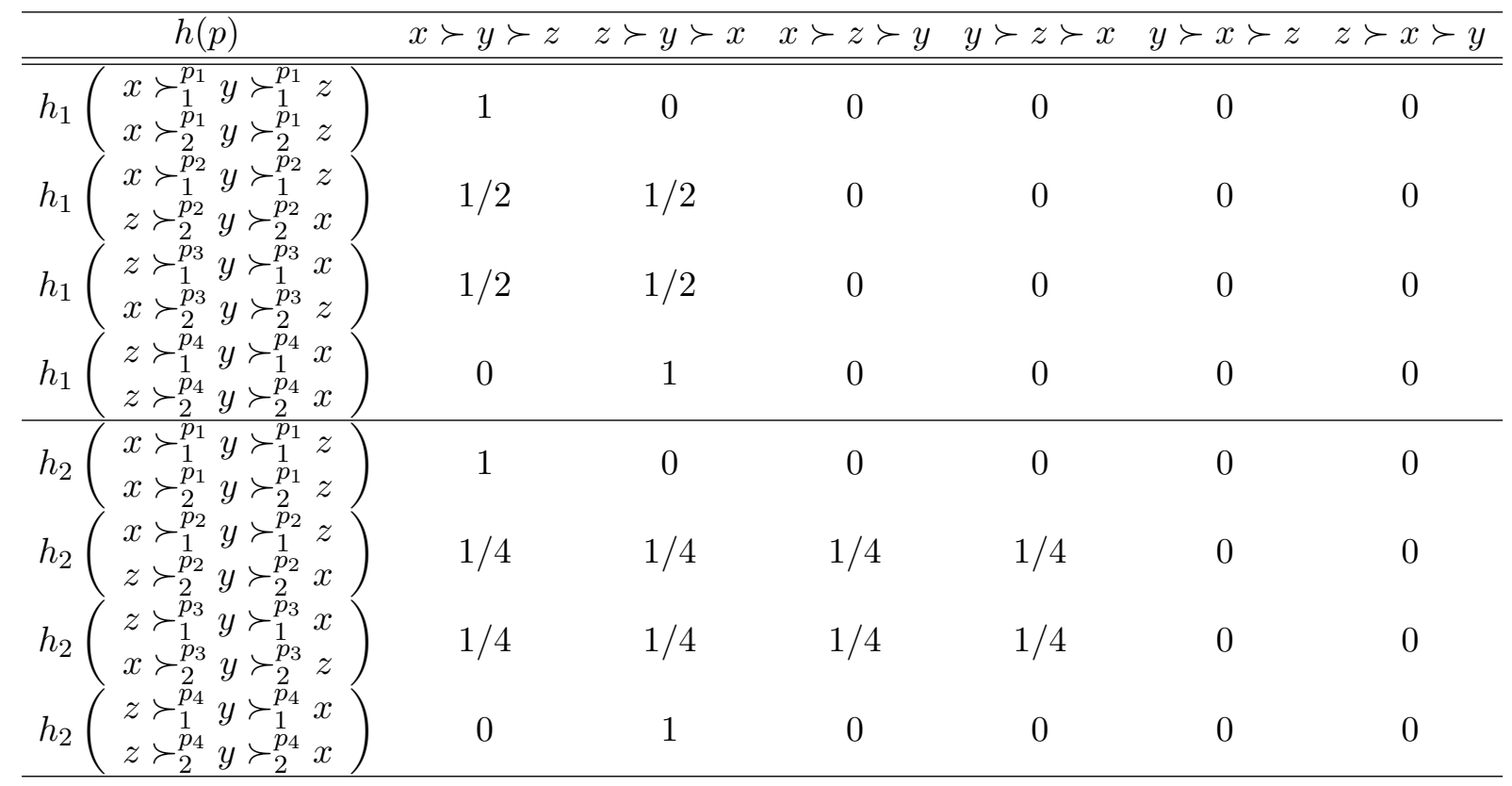

The following result shows that we have only to check the extreme points of the polytope when we judge whether $\Gamma^{\mathcal{P}}$ is surjective.

Theorem 5.9. The function $\Gamma^{\mathcal{P}}$ is surjective if and only if for each extreme point $d^{*}$ of $\mathcal{O}^{\mathcal{P}}$ there exists a probabilistic ASWF $h^{*}$ such that $\Gamma^{\mathcal{P}}\left(h^{*}\right)=d^{*}$.

Proof. The "only if" part is trivial, and we show the "if" part. For a given $\mathcal{P}$ we denote all the extreme points of polytope $\mathcal{O}^{\mathcal{P}}$ by $d_{1}^{*}, \ldots, d_{\mathcal{K}}^{*}$. Then for any point $\bar{d}$ in $\mathcal{O}^{\mathcal{P}}$ there is $\lambda_{1}, \ldots, \lambda_{\mathcal{K}}$ satisfying $\sum_{i=1}^{\mathcal{K}} \lambda_{i}=1$ as well as $\lambda_{i} \geq 0$ for each $i \in\{1, \ldots, \mathcal{K}\}$ and $\bar{d}$ can be written as

$$
\bar{d}=\sum_{i=1}^{\mathcal{K}} \lambda_{i} d_{i}^{*} .
$$

For each $i \in\{1, \ldots, \mathcal{K}\}$ let $h_{i}^{*}$ a probabilistic ASWF with $\Gamma^{\mathcal{P}}\left(h_{i}^{*}\right)=d_{i}^{*}$. We define $\bar{h}$ as

$$
\bar{h}(p)(\triangleright):=\sum_{i=1}^{\mathcal{K}} \lambda_{i} h_{i}^{*}(p)(\triangleright)
$$

for each $p \in \mathcal{P}$ and for each $\triangleright \in L(\mathcal{X})$, then $\bar{h}$ is a probabilistic ASWF and $\Gamma^{\mathcal{P}}(\bar{h})=\bar{d}$.

Let us consider the polytope determined by (5.8)-(5.10), (5.11) and the nonnegativity constraint of $\mu$. Combining (5.8) and (5.11), we have (5.9). Furthermore (5.10) is obtained from (5.11) and nonnegativity:

$$
\begin{aligned}
\mu(T)+\mu(S)-\mu(S \cup T) & =\mu(T)+\mu(S \cap T)+\mu(S \backslash T)-\mu(S \backslash T)-\mu(T) \\
& =\mu(S \cap T) \geq 0 .
\end{aligned}
$$


Thus (5.8)-(5.11) together with the nonnegativity constraint is equivalent to the system of (5.8), (5.11) and the nonnegativity. Therefore there is an $n$-dimensional vector $\pi=$ $\left(\pi_{1}, \ldots, \pi_{n}\right)$ in the regular simplex $\Pi:=\left\{\pi \in \mathbb{R}^{n} \mid \pi \geq 0 ; \sum_{i=1}^{n} \pi_{i}=1\right\}$ such that

$$
\mu(S)=\sum_{i \in S} \pi_{i}
$$

holds for every $S \subseteq \mathcal{N}$. Conversely, for a given $\pi \in \Pi, \mu(S):=\sum_{i \in S} \pi_{i}$ satisfies (5.8), (5.11) and nonnegativity. Therefore the polytope defined by (5.8)-(5.11) and the nonnegativity is the image of the regular simplex $\Pi \subseteq \mathbb{R}^{n}$ under the linear function defined by (5.12). Since each extreme point of the polytope is an image of an extreme point of $\Pi$, which is a unit vector, we see that all the extreme points of the polytope are integer vectors. In fact, for each $i \in \mathcal{N}$ let $\mu^{i} \in \mathbb{R}^{2^{\mathcal{N}}}$ be defined by

$$
\mu^{i}(S):= \begin{cases}1 & \text { if } i \in S \\ 0 & \text { otherwise }\end{cases}
$$

Then the set of extreme points of the polytope consists of $\mu^{i}$ 's for $i \in \mathcal{N}$. Since, as we have seen, an integer extreme point of the polytope is an image of an ASWF, this together with Theorem 5.9 means that a mapping from a probabilistic ASWF to the polytope determined by (5.8)-(5.11) and $\mu \geq 0$ via (5.7) is surjective.

\section{Concluding Remarks.}

This paper presented a study on the linear inequality representation of Arrovian Social Welfare Functions. We gave a proof of the ASWF integer linear inequality representation theorem of [12] by introducing the profile-dependent integer linear inequality representation of ASWFs. Our way of proof can be applied to derive another linear inequality formulation of ASWFs for different preference domain frameworks such as the one discussed in Ando et al. [1] and in Ohbo et al. [8] as well as an ASWF on weak orderings. See Sato [9] for further details. We also studied the polyhedral structure determined by the original ASWF linear inequalities on single-peaked domains, single-caved domains, and the domains where each triple of alternatives contains one that cannot be medium, showing that the set of nonnegative solutions to the inequalities forms an integral polytope when these domains satisfy weakly nonisolated condition. We then showed that a real vector satisfying the linear inequalities can be created from any probabilistic ASWF and derived the subadditive function of [4] as a special case. We also considered the construction of a probabilistic ASWF from a given nonnegative solution to the inequalities.

There still remain interesting problems unsolved. One is the characterization of social choice functions as a system of inequalities. Our counter-example to [12] indicates that necessary conditions to form an integral polytope on single-peaked domains are still open. A construction problem of a probabilistic ASWF from a solution to the linear inequalities on restricted domains is worth further study. Study of the probabilistic version of social choice functions in Gibbard [5] through inequality representations is also left for future research.

\section{Acknowledgments}

The authors thank anonymous referees for their valuable comments. They also thank René van den Brink and Gerard van der Laan, Vrije Universiteit, Amsterdam, the Netherlands, for stimulating discussion. The second author is supported by the grant B 46-551 
from the Netherlands Organisation for Scientific Research, and also by the Grant-in-Aid for Scientific Research (B) 18310101 of the Ministry of Education, Culture, Sports, Science and Technology of Japan.

\section{References}

[1] K. Ando, A. Ohara, and Y. Yamamoto: Impossibility theorems on mutual evaluation. Journal of the Operations Research Society of Japan, 46 (2003), 523-532 (in Japanese).

[2] K.J. Arrow: Social Choice and Individual Values, 2nd edition (Wiley, New York, 1963).

[3] K.J. Arrow, A.K. Sen, and K. Suzumura (eds.): Handbook of Social Choice and Welfare, volume 1 (Elsevier, Amsterdam, 2002).

[4] S. Barberá and H. Sonnenschein: Preference aggregation with randomized social orderings. Journal of Economic Theory, 18 (1978), 244-254.

[5] A. Gibbard: Manipulation of schemes that mix voting with chance. Econometrica, 45 (1977), 665-681.

[6] E. Kalai and E. Muller: Characterization of domains admitting nondictatorial social welfare functions and nonmanipulable voting procedures. Journal of Economic Theory, 16 (1977), 457-469.

[7] A. McLennan: Randomized preference aggregation: Additivity of power and strategy proofness. Journal of Economic Theory, 22 (1980), 1-11.

[8] K. Ohbo, M. Tsurutani, M. Umezawa, and Y. Yamamoto: Social welfare function for restricted individual preference. Pacific Journal of Optimization, 1 (2005), 315-325.

[9] K. Sato: A Study on Linear Inequality Representation of Social Welfare Functions (Master's thesis, Graduate School of Systems and Information Engineering, University of Tsukuba, 2006).

[10] A.K. Sen: A possibility theorem on majority decisions. Econometrica, 34 (1966), 491-499.

[11] A.K. Sen: Collective Choice and Social Welfare (Holden-Day, San Francisco, 1970).

[12] J. Sethuraman, C.P. Teo, and R.V. Vohra: Integer programming and Arrovian social welfare functions. Mathematics of Operations Research, 28 (2003), 309-326.

Keisuke Sato

Transport Information Technology Division Railway Technical Research Institute 2-8-38 Hikari-cho, Kokubunji-shi

Tokyo 185-8540, Japan

E-mail: keisato@rtri.or.jp 